\title{
Quantifying dynamics of the financial correlations
}

\author{
S. Drożdż ${ }^{\mathrm{a}, \mathrm{b}}$, J. Kwapień ${ }^{\mathrm{a}}$, F. Grümmer ${ }^{\mathrm{b}}$, F. Ruf ${ }^{\mathrm{c}}$, J. Speth ${ }^{\mathrm{b}, \mathrm{d}}$ \\ a Institute of Nuclear Physics, Radzikowskiego 152, 31-342 Kraków, Poland \\ ${ }^{\mathrm{b}}$ Institute für Kernphysik, Forschungszentrum Jülich, D-52425 Jülich, Germany \\ ${ }^{\mathrm{c}}$ West LB International S.A., 32-34 bd Grande-Duchesse Charlotte, L-2014 \\ Luxembourg \\ ${ }^{\mathrm{d}}$ Centre for the Subatomic Structure of Matter, University of Adelaide, SA 5005, \\ Australia
}

\begin{abstract}
A novel application of the correlation matrix formalism to study dynamics of the financial evolution is presented. This formalism allows to quantify the memory effects as well as some potential repeatable intradaily structures in the financial time-series. The present study is based on the high-frequency Deutsche Aktienindex (DAX) data over the time-period between November 1997 and December 1999 and demonstrates a power of the method. In this way two significant new aspects of the DAX evolution are identified: (i) the memory effects turn out to be sizably shorter than what the standard autocorrelation function analysis seems to indicate and (ii) there exist short term repeatable structures in fluctuations that are governed by a distinct dynamics. The former of these results may provide an argument in favour of the market efficiency while the later one may indicate origin of the difficulty in reaching a Gaussian limit, expected from the central limit theorem, in the distribution of returns on longer time-horizons.
\end{abstract}

\section{Introduction}

In global terms the financial correlations can be classified as correlations in space and correlations in time, though of course they are somewhat interrelated. The first category so far studied involves for instance the correlations among the companies comprised by a single stock market [1-3], among a group of subjects $[4,5]$ and even between the different and geographically remote stock markets [6]. From practical perspective this type of correlations relates to the theory of optimal portfolios $[7,8]$ and risk management. In the context of the stock market an important result of this study is that majority

Preprint submitted to Elsevier Preprint 27 October 2018 
of eigenvalues in the spectrum of the correlation matrix agree very well with the universal predictions of random matrix theory $[9,10]$. Locations of some of the eigenvalues differ however from these predictions and thus, similarly as in other physical systems [11,12], identify certain system-specific, non-random properties such as collectivity.

Studying explicit correlations in time is at least as important because it is this type of correlations that directly reflects a nature of the financial dynamics. In general the character of those correlations is however less understood and several related issues still remain puzzling. It is for instance commonly accepted [13] that the autocorrelation function of the financial time-series drops down to zero within few minutes which reflects a time-horizon of the market inefficiency. At the same time the correlations in volatility remain positive for many weeks. On short time-scales the return distributions are definitely not Levy stable [14] but it turns out difficult to detect their convergence to a Gaussian on longer time-scales as expected from the central limit theorem. In this connection it is also appropriate to mention a still poorly understood phenomenon of log-periodicity $[15,16]$ which seems to reflect existence of some very specific correlations on all time-scales. As a contribution towards resolving this sort of difficulties below we propose to use the concept of the correlation matrix such that it focuses entirely on the time-correlations and their potential existence can parallelly be detected on various time-scales. Utility of such a procedure is illustrated on an example of high-frequency (15sec) recordings of the Deutsche Aktienindex (DAX). The entries of the correlation matrix are then constructed from the time-series of returns representing the consecutive trading days. As a result such entries are labelled by the pairs of different days. Several striking observations are also made.

\section{Methodology}

The relevant correlation matrix is defined as follows. To each element in a certain sequence $N$ of relatively long consecutive time-intervals of equal length $T^{\prime}$ labelled with $\alpha$ one uniquely assigns a time series $x_{\alpha}\left(t_{i}\right)$, where $t_{i}(i=$ $\left.1, \ldots, T^{\prime}\right)$ is to be understood as discrete time counted from the beginning for each $\alpha$. In the present case $\alpha$ is going to label the consecutive trading days and $t_{i}$ the trading time during the day. Similar methodology has already been successfully applied [17] to extract the repeatable structures in the brain sensory response.

If, as here, $x_{\alpha}\left(t_{i}\right)$ represents a price time-series than it is natural to define the 
returns $G_{\alpha}\left(t_{i}\right)$ time-series as

$$
G_{\alpha}\left(t_{i}\right)=\ln x_{\alpha}\left(t_{i}+\tau\right)-\ln x_{\alpha}\left(t_{i}\right) \simeq \frac{x_{\alpha}\left(t_{i}+\tau\right)-x_{\alpha}\left(t_{i}\right)}{x_{\alpha}\left(t_{i}\right)},
$$

where $\tau$ is the time lag. The normalized returns, with the average value subtracted and its variance normalized to unity, are defined by

$$
g_{\alpha}\left(t_{i}\right)=\frac{G_{\alpha}\left(t_{i}\right)-\left\langle G_{\alpha}\left(t_{i}\right)\right\rangle_{t}}{v^{2}}, \quad v=\sigma\left(G_{\alpha}\right)=\sqrt{\left\langle G_{\alpha}^{2}(t)\right\rangle_{t}-\left\langle G_{\alpha}(t)\right\rangle_{t}^{2}},
$$

where $v$ is volatility of $G_{\alpha}(t)$ and $\langle\ldots\rangle_{t}$ denotes averaging over time. One thus obtains $N$ time series $g_{\alpha}\left(t_{i}\right)$ of length $T\left(T=T^{\prime}-1\right)$, i.e. an $N \times T$ matrix $\mathbf{M}$. Then, the correlation matrix is defined as $\mathbf{C}=(1 / T) \mathbf{M M}^{\mathbf{T}}$. By diagonalizing C

$$
\mathbf{C} \mathbf{v}^{k}=\lambda_{k} \mathbf{v}^{k}
$$

one obtains the eigenvalues $\lambda_{k}(k=1, \ldots, N)$ and the corresponding eigenvectors $\mathbf{v}^{k}=\left\{v_{\alpha}^{k}\right\}$.

In the limiting case of entirely random correlations the density of eigenvalues $\rho_{C}(\lambda)$ defined as

$$
\rho_{C}(\lambda)=\frac{1}{N} \frac{d n(\lambda)}{d \lambda}
$$

where $n(\lambda)$ is the number of eigenvalues of $\mathbf{C}$ less than $\lambda$, is known analytically [18], and reads

$$
\begin{array}{r}
\rho_{C}(\lambda)=\frac{Q}{2 \pi \sigma^{2}} \frac{\sqrt{\left(\lambda_{\max }-\lambda\right)\left(\lambda-\lambda_{\min }\right.}}{\lambda} \\
\lambda_{\min }^{\max }=\sigma^{2}(1+1 / Q \pm 2 \sqrt{1 / Q})
\end{array}
$$

with $\lambda_{\min } \leq \lambda \leq \lambda_{\max }, Q=T / N \geq 1$, and where $\sigma^{2}$ is equal to the variance of the time series (unity in our case).

\section{Results}

Our exploratory study along the above indicated line is based on the DAX recordings with the frequency of $15 \mathrm{sec}$ during the period between November 

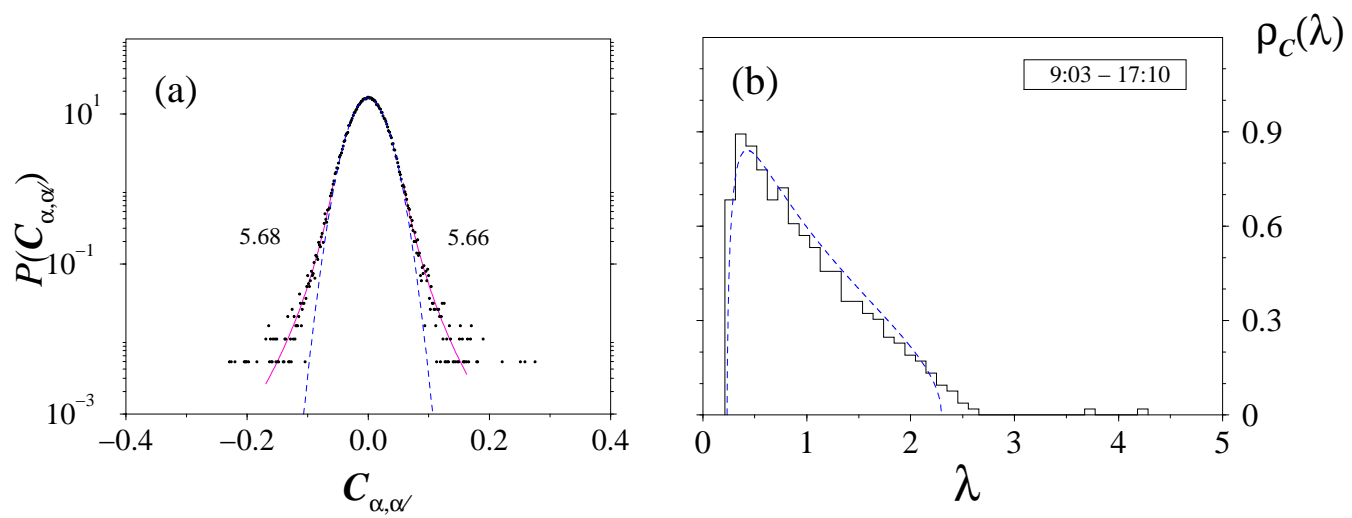

Fig. 1. $517 \times 517$ correlation matrix $\mathbf{C}$ calculated from the 15 sec frequency DAX variation during 9:03 - 17:10 intraday trading time. $N=517$ is the number of trading days (labelled by $\alpha$ ) qualified for this study from the calendar period November 28th, 1997 - December 30th, 1999. The dashed line represents the best fit in terms of a Gaussian while the solid line indicates a power law fit to the tails of this distribution. The numbers reflect the corresponding scaling indices. (b) The probability density (histogram) of the eigenvalues of $\mathbf{C}$ and the corresponding null hypothesis (dashed line) formulated in terms of eq. (4).

28th, 1997 and December 30th, 1999. By taking the DAX intraday variation between the trading time 9:03 and 17:10 which corresponds to $T=1948$ one then obtains $N=517$ complete and equivalent time series representing different trading days during this calendar period. (Several days with incomplete recordings have been rejected.) Using this set of data we construct the $517 \times 517$ matrix C.

A characteristics of primary interest is the structure of eigenspectrum of $\mathbf{C}$. The structure of eigenspectrum of a matrix is expected to be related $[11,12]$ to the distribution of its elements. The distribution of such elements of $\mathbf{C}$ corresponding to the above specified procedure and the data set is shown in the left panel of Fig. 1 Clearly, this distribution is symmetric with respect to zero, a Gaussian like (dashed line) on the level of small matrix elements, but sizably thicker than a Gaussian on the level of large matrix elements. As indicated in the figure, in the latter case, a power law with the index of about 5.6 (consistent with the distribution of returns) provides a reasonable representation. This is far beyond the Levy stable regime but points to the two ensembles of random matrices, the Gaussian $[9,10,18]$ and the Levy distributed [19,20], as interesting limits for formulating the null hypotheses.

The resulting probability density of eigenvalues, shown in the right panel of Fig. 1, displays a somewhat unexpected structure. There exist two outliers significantly above $\lambda_{\max }$ (for $Q=1948 / 517, \lambda_{\max } \approx 2.3$ ) which seem to indicate that the dynamics under consideration is not a pure noise, but instead that certain time specific repeatable structures in the intraday trading do take place. However, it is even more astonishing that the bulk of the spectrum agrees 

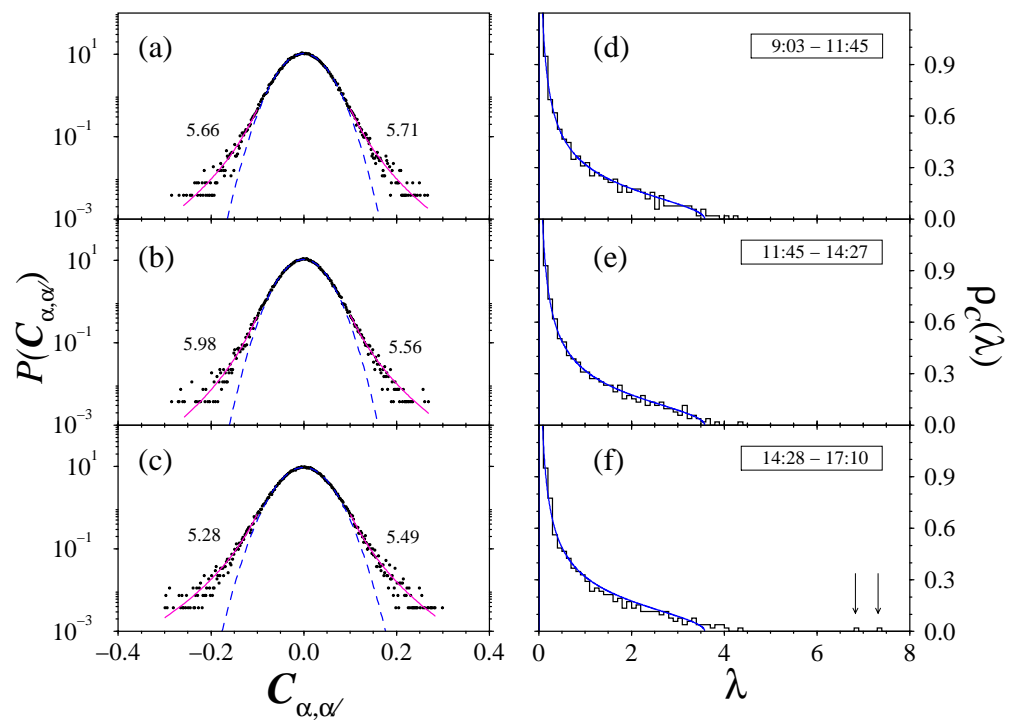

Fig. 2. (Left column) Distribution of matrix elements $C_{\alpha, \alpha^{\prime}}$ of the $517 \times 517$ correlation matrix $\mathbf{C}$ calculated from the 15 sec frequency DAX variation during the intraday trading time 9:03-11:45 (a), 11:45-14:28 (b) and 14:28-17:10 (c). Similarly as in Fig. $1 N=517$ is the number of trading days (labelled by $\alpha$ ) qualified for this study from the calendar period November 28th, 1997 - December 30th, 1999. The solid lines indicate the power law fits to the tails of these distributions, while the dashed one corresponds to a Gaussian best fit. The numbers reflect the corresponding scaling indices. (Right column) The probability densities (histograms) of the eigenvalues of $\mathbf{C}(\mathrm{d})$, (e) and (f) corresponding to the three (a), (b) and (c) cases, respectively. Their null hypotheses formulated in terms of eq. (4) are indicated by the continuous lines.

very well with the bounds prescribed by purely random correlations. This indicates that the neighbouring recordings in our time series of 15 sec DAX returns share essentially no common information. Our previous experience [17] teaches us that the probability density of eigenvalues of the correlation matrix is a sensitive measure of such effects. Typically, a common information shared by neighbouring events results in an effective number $\left(T_{e f f}\right)$ of time points such that $T_{\text {eff }}$ is significantly smaller than $T$. This immediately affects both $\lambda_{\max }$ and $\lambda_{\min }$. In the present case we see basically no such effect, particularly on $\lambda_{\min }(\approx 0.23)$ side. A whole nonrandomness can be here associated with the two largest eigenvalues.

In order to identify the character of eigenstates associated with these eigenvalues we split the whole daily time interval considered into three equal subintervals. For each of them $T=649$ (thus $\lambda_{\min } \approx 0.01$ and $\lambda_{\max } \approx 3.58$ ). The resulting distributions of matrix elements of $\mathbf{C}$ and the corresponding probability densities of eigenvalues are shown in Fig. 2. It appears easy to see that separation of the two largest eigenvalues originates from the last subinterval. Consistently, it is also this subinterval which produces the thickest tails in the distribution of large matrix elements. 


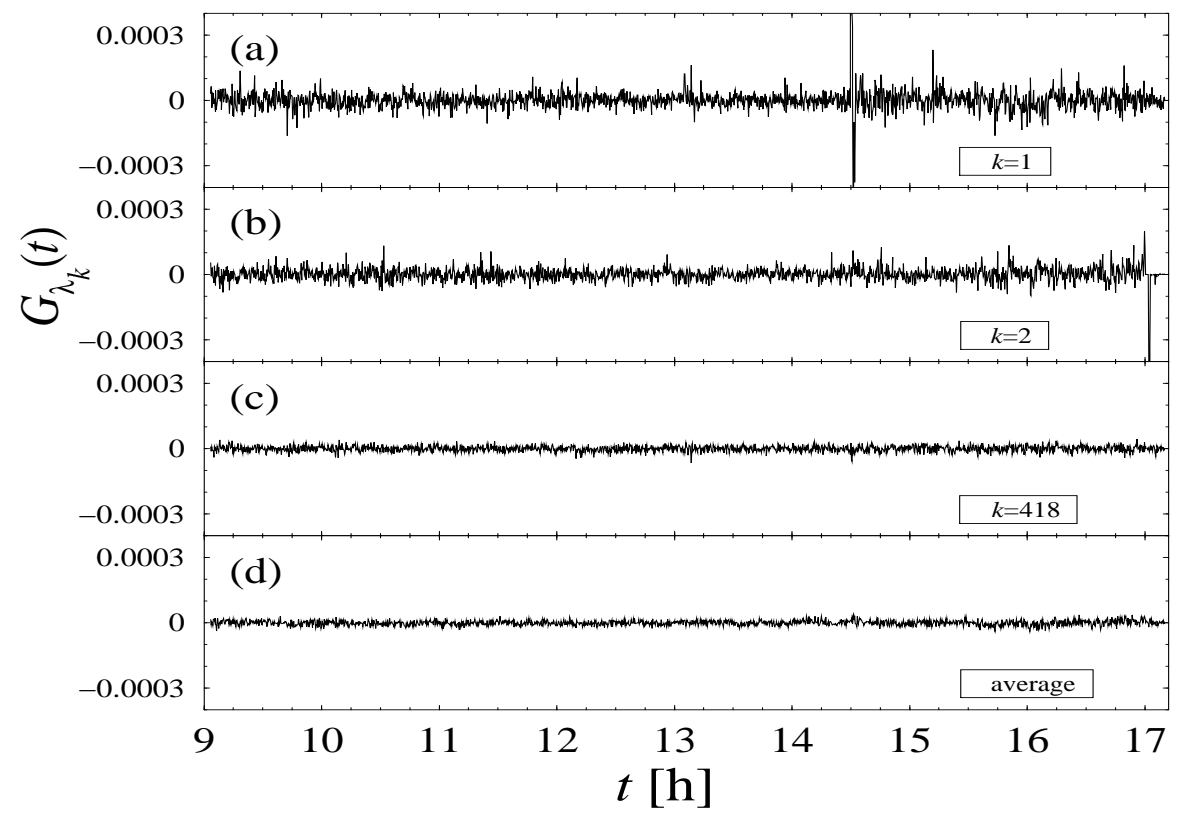

Fig. 3. The superposed time series of unnormalized returns calculated according to eq. (6) for $k=1$ (a), $k=2$ (b) and $k=418$ (c), and of the simple average (d) of the original returns as functions of the intraday trading time.

An even more efficient way to visualize the differences among the eigenvectors is to look at the superposed time series of returns

$$
G_{\lambda_{k}}\left(t_{i}\right)=\sum_{\alpha=1}^{N} \operatorname{sign}\left(v_{\alpha}^{k}\right)\left|v_{\alpha}^{k}\right|^{2} G_{\alpha}\left(t_{i}\right) .
$$

In this definition $\left|v_{\alpha}^{k}\right|^{2}$ is used instead of $v_{\alpha}^{k}$ for the reason of preserving normalization and the sign of $v_{\alpha}^{k}$ to account for a possible coherence of the original signals. A collection of such superposed time series of returns for $k=1,2$ and 418 , and of the simple average of the original returns is shown in Fig. 3. For a statistical value of $k$, as it is illustrated by an example of $k=418$ ((c) in Fig 3), $g_{\lambda_{k}}\left(t_{i}\right)$ basically does not differ from the simple average (panel (d) in Fig. 3). The first two differ however significantly and indicate the existence of repeatable structures at the well defined instants of time through many days. Quite unexpectedly, the most collective signal, associated with the largest eigenvalue ( $k=1$ shown in (a) of Fig. 3), reflects the strongest synchronous DAX activity precisely at around 14:30 (probably in response to the North-American financial news release exactly at this time) and not at the time when the Wall Street opens, nor even just before closing in Frankfurt. This last period of an enhanced activity also carries some signatures of synchrony but is entirely comprised by the second eigenstate.

Another characteristics which carries the relevant information is the probability distribution of the eigenvector components $v_{\alpha}^{k}$. Such a probability distribu- 


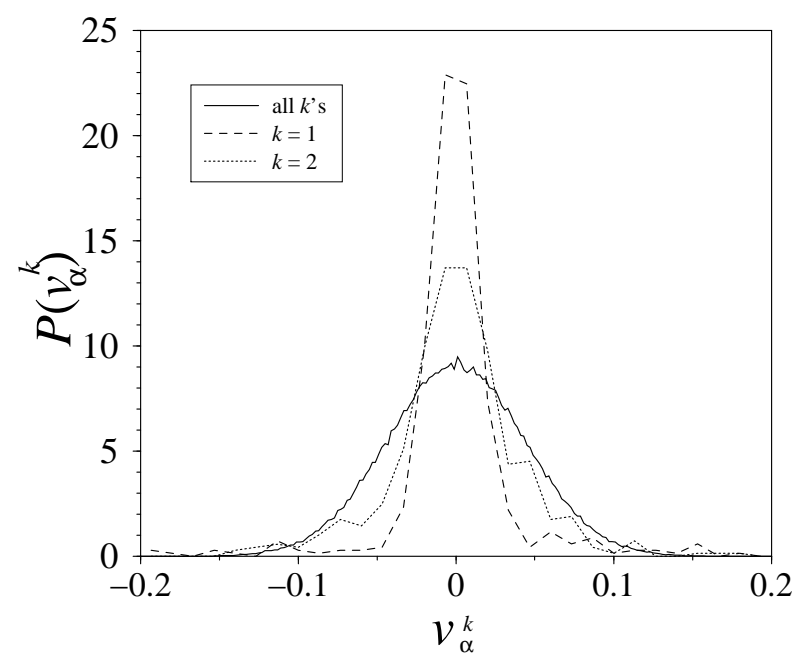

Fig. 4. Distribution of eigenvector components $v_{\alpha}^{k}$ for the two most collective eigenstates $(k=1$ and $k=2)$ together with the corresponding distribution of components for all the eigenstates.

tion evaluated from all $k$ 's is illustrated by the solid line in Fig. 4 . It globally is well represented by a Gaussian. Significantly different are the distributions of eigenvector components for our two outliers; they are concentrated more at around zero but, at the same time, the tails of the distributions are thicker. This effect is more pronounced for $k=1$ than for $k=2$ which indicates that fewer days $(\alpha$ 's) contribute to the strong signal seen at 14:30 for $k=1$ than just after 17:00 for $k=2$.

This issue is quantified in more detail in Fig. 5 which shows how these two eigenvector components are distributed in magnitude over the trading days ( $\alpha$ 's) incorporated in the present study. For $k=1$ such components assume large values indeed and even seem to be developing certain periodicity, which in the initial period considered here corresponds to about 20 trading days (about one calendar month). Interestingly, it is this period which corresponds to our classic example of "Imprints of log-periodic self-similarity in the stock market" [16]. Perhaps the observed, equidistant in time, synchronous bursts of activity constitute one of the elements introducing a characteristic time-scale responsible for discrete scale invariance [21], an element which in natural way may contribute to the appearance of the log-periodic oscillations.

Finally, we find it appropriate to notice that the return probability density distributions associated with the above identified synchronous bursts of activity seem to be governed by a different law than what more global statistical analyses [22] document. Fig. 6 shows the tails of the return $G_{\alpha}\left(t_{i}\right)$ distribution (triangles) for the time period 14:25-14:35 through all the DAX 517 trading days considered here. The statistics is of course poor but still it quite convincingly indicates much thicker tails as compared to the periods of 'normal' activity, represented in this figure (circles) by the period 9:03-14:25. In the 


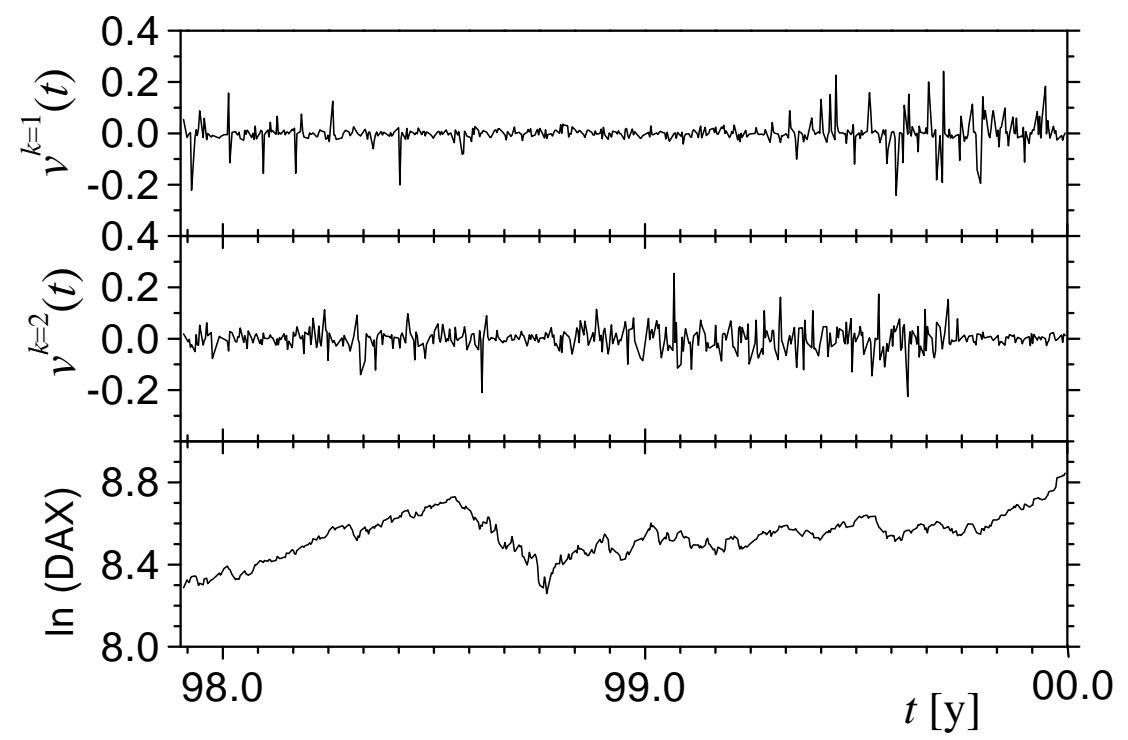

Fig. 5. Eigenvector components $v_{\alpha}^{k}$ for $k=1$ (upper) and $k=2$ (middle) as a function of trading days from the entire period November 28th, 1997 - December 30th, 1999. For a comparison, the logarithm of DAX time variation is presented in the bottom panel.

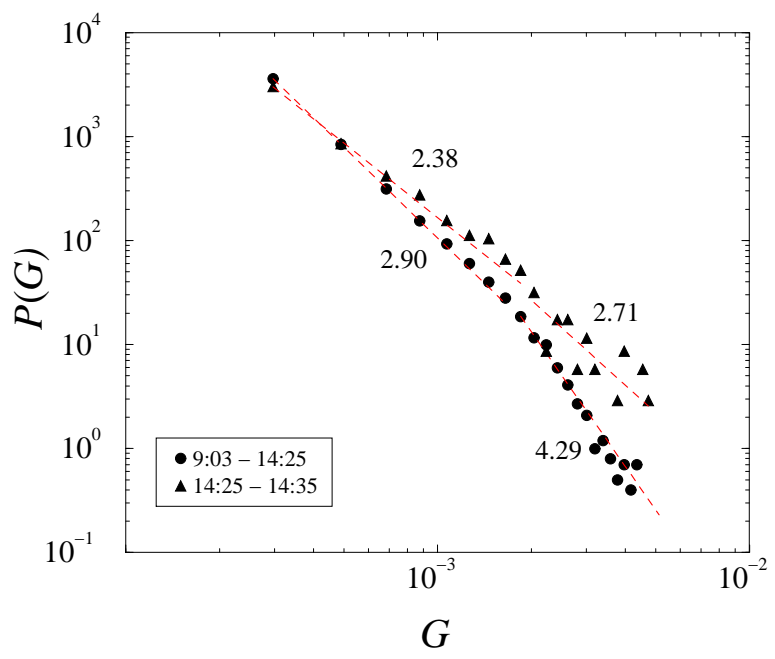

Fig. 6. Tails of the return distribution through all 517 trading days for the periods 9:03-14:25 and 14:25-14:35. The corresponding linear best fits indicate (see also the corresponding numbers) two different regimes (dashed lines).

latter case the pdf parameters are about consistent with those cited in the literature [22], while in the short time interval at around 14:30 we seem to be still in the Lévy stable regime. 


\section{Summary}

The present study sheds a somewhat new light on the issue of time-correlations in the dynamics of financial evolution. It shows for instance that the consecutive returns carry essentially no common information even when probed with the frequency of $15 \mathrm{sec}$. Such a conclusion sounds somewhat in contrast to a common belief, based on autocorrelation function analysis, that the market inefficiency time-horizon extends over few minutes. There is however perhaps no contradiction between our conclusion and the time-lag dependence of the autocorrelation function. This function remains positive for about few minutes indeed, but already after $15 \mathrm{sec}$ it drops down by at least one order of magnitude. On the other hand, however, at the well defined short periods of time during the intraday trading, there exist clearly identifiable synchronous repeatable bursts of activity (for DAX at 14:30) whose related return probability density functional develops significantly larger values on the level of rare events than during periods of the normal activity. This, together with the fact that such events are strongly correlated in time, may constitute a principal reason for an observed extremely slow convergence to a Gaussian of the global return distribution on long-time scales. It would now be very interesting to perform similar study for the other stock market indices as well.

\section{References}

[1] L. Laloux, P. Cizeau, J-.P Bouchaud, M. Potters, Phys. Rev. Lett. 83 (1999) 1467.

[2] V. Plerou, P. Gopikrishnan, B. Rosenow, L.A.N. Amaral, H.E. Stanley, Phys. Rev. Lett. 83 (1999) 1471.

[3] S. Drożdż, F. Grümmer, A.Z. Górski, F. Ruf, J. Speth, Physica A 287 (2000) 440

[4] R.N. Mantegna, Eur. Phys. J. B 11 (1999) 193

[5] L. Kullmann, J. Kertész, R.N. Mantegna, Physica A 287 (2000) 412

[6] S. Drożdż, F. Grümmer, F. Ruf, J. Speth, Towards identifying the world stock market cross-correlations: DAX versus Dow Jones, arXiv:cond-mat/0011488, Physica A in press

[7] H. Markowitz, Portfolio Selection: Efficient Diversification of Investments, J. Wiley and Sons, New York, 1959

[8] E.J. Elton and M.J. Gruber, Modern Portfolio Theory and Investment Analysis, J. Wiley and Sons, New York, 1995 
[9] E.P. Wigner, Ann. Math. 53 (1951) 36.

[10] M.L. Mehta, Random Matrices, Academic Press, Boston, 1999

[11] S. Drożdż, S. Nishizaki, J. Speth, M. Wójcik, Phys. Rev. E 57 (1998) 4016.

[12] S. Drożdż, M. Wójcik, On the origin of order from random two-body interactions, arXiv:nucl-th/0007045

[13] J.Y. Campbell, A.W. Lo, A. Craig MacKinley, The Econometrics of Financial Markets, Princeton University Press, Princeton, NJ, 1997

[14] H.E. Stanley, P. Gopikrishnan, V. Plerou, L.A.N. Amaral, Physica A 287 (2000) 339

[15] D. Sornette, A. Johansen, J.-P. Bouchaud, J. Phys. I France 6 (1996) 167;

J.A. Feigenbaum, P.G.O. Freund, Int. J. Mod. Phys. B 10 (1996) 3737

[16] S. Drożdż, F. Ruf, J. Speth and M. Wójcik, Eur. Phys. J. B10 (1999) 589.

[17] J. Kwapień, S. Drożdż, A.A. Ioannides, Phys. Rev. E62 (2000) 5557

[18] A. Edelman, SIAM J. Matrix Anal. Appl. 9 (1988) 543;

A.M. Sengupta, P.P. Mitra, Phys. Rev. E 60 (1999) 3389

[19] P. Cizeau, J.-P. Bouchaud, Phys. Rev. E 57 (1994) 1810

[20] Z. Burda, R.A. Janik, J. Jurkiewicz, M.A. Nowak, G. Papp, I. Zahed, Free Random Levy Matrices, arXiv:cond-mat/0011451

[21] D. Sornette, Phys. Rep. 297 (1998) 239

[22] R.N. Mantegna, H. Eugene Stanley, An Introduction to Econophysics: Correlations and Complexity in Finance, University Press, Cambridge, 2000 